\title{
Understanding and quantifying the immune microenvironment in hepatocellular carcinoma
}

\author{
Juan C. Osorio ${ }^{1,2}$, James J. Harding ${ }^{1,2}$ \\ ${ }^{1}$ Gastrointestinal Oncology Service, Department of Medicine, Memorial Sloan Kettering Cancer Center, New York, NY, USA; ${ }^{2}$ Weill Cornell \\ Medical College, New York, NY, USA \\ Correspondence to: James J. Harding. 300 East 66th Street, New York, NY 10065, USA. Email: hardinj1@mskcc.org. \\ Provenance: This is an invited Editorial commissioned by Section Editor Rui Liao (Department of Hepatobiliary Surgery, The First Affiliated \\ Hospital of Chongqing Medical University, Chongqing, China). \\ Comment on: Kim HD, Song GW, Park S, et al. Association Between Expression Level of PD1 by Tumor-Infiltrating CD8+ T Cells and Features of \\ Hepatocellular Carcinoma. Gastroenterology 2018;155:1936-50.e17.
}

Received: 30 November 2018; Accepted: 05 December 2018; Published: 24 December 2018.

doi: $10.21037 /$ tgh.2018.12.04

View this article at: http://dx.doi.org/10.21037/tgh.2018.12.04

Kim and colleagues reported recently on the molecular and functional phenotypes of tumor-infiltrating lymphocytes (TILs) derived from a cohort of patients with early stage hepatocellular carcinoma (HCC) (1). Briefly, cytometric methods were employed to isolate and to interrogate TILs and hepatic lymphocytes from 90 surgicallyresected specimens from a single academic center. The authors proposed that TILs are subdivided based on the level of expression of program death receptor-1 (PD1): PD1-high, PD1-intermediate, and PD1-low/negative. Using RNA sequencing on sorted TILs, each subpopulation exhibited a distinct gene expression profile with the PD1-high CD8 ${ }^{+} \mathrm{T}$ cell subset expressing genes that impair T-cell cytokine secretion and proliferation. PD1-high TILs also co-expressed other immune checkpoint molecules such as TIM3 and LAG3, and when compared to PD1intermediate and PD1-negative subsets, produced lower amounts of TNF and IFN- $\gamma$ upon in vitro T-cell receptor stimulation. This T-cell exhaustion phenotype, which was evident in about half of the cohort, was associated with aggressive disease features (i.e., larger tumor burden, higher alpha-fetoprotein levels, and greater proportion with vascular invasion) and a biomarker profile that predicted response to immune checkpoint inhibitors (e.g., tumoral PD1 expression by immunohistochemistry and T-cell gene expression signatures). Furthermore, a correlation between TILs and circulating PD1 high CD8 ${ }^{+} \mathrm{T}$ cells was apparent; suggesting that peripheral blood sampling might have some ability to monitor the tumoral immune microenvironment. Finally, to assess the therapeutic relevance of their findings, the authors conducted in vitro assays demonstrating that dual blockade of PD1 plus TIM3 or LAG3 restored T cell activity in PD1-high TILs, while it did not promote similar responses in PD1-low TILs.

The authors should be applauded for a well-written and well-designed retrospective cohort study, which represents an important translational undertaking in HCC (1). The report highlights the cumulative interest in understanding the immune microenvironment in cancer and brings up several critical, and yet, partially or unanswered questions. It is clear that subsets of HCC induce an immune response, however parsing out and characterizing the types of immune responses are an area of active investigation. These investigations have become especially relevant now that anti-PD1 monoclonal antibodies have led to rapid, deep, and durable responses in $16-20 \%$ of patients with advanced HCC (2-4). Key questions include what subset of HCCs elicit an effective immune response; how immune responses to HCCs should be measured; and what clinical implications, if any, does the level of immune activation have for potent immunotherapy responses. As a major hypothesis is that immunologically active tumors, specifically those with an exhausted T-cell phenotype, should respond to immune checkpoint inhibitors, it is imperative to develop validated clinical grading methods to assess the degree of anti-tumor immune response in an individual patient. 
Acknowledging the limitations of a small retrospective cohort study conducted at one center, the work of Kim and colleagues is consistent with several recent publications indicating that an HCC subset (at least $20 \%)$ engenders a brisk immune response $(1,5-7)$. As illustrated nicely in their work, the immunologic milieu in HCC is diverse, and at least dichotomous in tumors that illicit an immune response, with either an active or exhausted immune phenotype $(1,7)$. Further highlighting the immense complexity of characterizing the immune microenvironment, a recent report identified $11 \mathrm{~T}$-cell subsets, each with unique functional properties, from 6 HCC patients using single cell sequencing (6). Antitumor immune responses are dynamic processes and therefore, absolute immune-based biomarkers are difficult to develop and standardize. For example, static assays such as the presence or absence of TILs or PD-L1 expression would be an inadequate method to identify patients who are apt to respond to immune checkpoint inhibitors. This is reflected in more simplistic approaches such as PD-L1 immunohistochemistry, where several different assays and cut-off criteria had been proposed to determine positivity $(3,8)$. PD-L1 testing is highly dependent on the tumor type (9), the tissue quality, tumoral heterogeneity, the percentage positivity threshold, and the antibodies used for PD-L1 detection (10). In the clinic, pretreatment PD-L1 status is not associated with response to nivolumab in patients with advanced HCC (3).

Interrogation of the tumor with multiplex immunohistochemistry, T-cell gene expression profiles, and flow cytometric methods (such as those employed by the authors) are some of the current strategies used to overcome this limitation $(2,11)$. Application of flow cytometry in the peripheral blood would also have clear clinical import, and it is encouraging that Kim and colleagues saw a preliminary correlation between peripheral blood PD1-expressing total and TAA-specific $\mathrm{CD} 8^{+} \mathrm{T}$ cells and percentages of $\mathrm{PD}^{+}$ tumor-infiltrating $\mathrm{CD}^{+} \mathrm{T}$ cells, particularly with $\mathrm{PD} 1$-high TILs. Functional T-cell imaging is also in development to help define an ex vivo marker of immune activation (e.g., 89Zr-Df-IAB22M2C, NCT03107663). Larger or pooled series of more diverse HCC patient populations will be required to understand how the immune microenvironment is altered by ethnic diversity, etiological factors, tumoral stage, and sequential regional and systemic therapies as well as to further validate these proposed biomarkers of response to immune checkpoint inhibitors.

In parallel to these efforts to quantify the immune microenvironment, tumoral and host factors may regulate the immunologic response and may also have value as predictive biomarkers to immune checkpoint inhibitors. An emerging variant of responsiveness to immune checkpoint blockade appears to be the mutational burden of certain cancer types (12-14). As HCC has a low-to-moderate mutational burden and rarely exhibits hypermutation (only 1 of 90 patients in this series had a microsatellite instability-high phenotype), we agree with the authors that mutational burden is unlikely to be the major determinant of responsiveness to immune checkpoint inhibitors in this disease (15). Alternatively, tumor specific factors such as viral particles or WNT pathway activation $(5,15)$ and host factors such as HLA type (16) should be evaluated in HCC. For example, several groups have shown that WNT pathway activation leads to T-cell exclusion, and in HCC (7), activating CTNNB1 mutations negatively correlate with gene expression profiles of immune activation. Furthermore, preliminary clinical data suggest that mutations that activate the WNT- $\beta$ catenin pathway are associated with innate resistance to immune checkpoint inhibitors (15). Although the relationship between tumoral specific factors and immune response was not elucidated by Kim and colleagues, several groups are now applying a multi-“omic" approach to layer genomic, epigenomic, and transcriptomic profiling of HCC and explore how tumoral specific factors correlate with their specific immunophenotype, and hence their response to immunotherapy.

Beyond the potential role of PD1-high T-cell subsets as enumerated by flow cytometry as a predictive biomarker, the work of Kim and colleagues contributes to the growing literature indicating that other immune checkpoint molecules (e.g., CTLA-4, TIM3, LAG3), either alone or in combination with PD1/PD-L1 antibodies, may have therapeutic merit in HCC (17-19). These preclinical findings support the rationale of implementing strategies that simultaneously target multiple mechanisms of T-cell dysfunction to reinvigorate the antitumor response. The findings that only the PD1-high subset exhibited additive benefit are noteworthy and suggests that combination of immune-based treatment is unlikely to be beneficially to all patients with HCC. Thus, this observation further highlights the need for biomarker development in this therapeutic space. Currently, several clinical trials are testing the efficacy of anti-PD-1 or PD-L1 monoclonal antibodies in combination with inhibitors that target key regulators of T-cell exhaustion including other checkpoint molecules (CTLA-4, LAG3, TIM3); soluble immunemediators (IDO, IL10, or TGF- $\beta$ ); and other cells in the 
tumor microenvironment (regulatory T cells or myeloidderived suppressor cells) (20). Furthermore, other rational strategies seek to pair immune checkpoint inhibitors with tyrosine kinase inhibitors, monoclonal antibodies to VEGF/ VEGFR, as well as regional treatments in efforts to augment response rates and improve patient outcomes $(21,22)$.

In summary, the work of Kim and colleagues represents an early attempt at understanding and measuring the immune environment in HCC, with an aim of developing predictive biomarkers to immune checkpoint inhibitors. Our expectation is that such works will dominate the academic press in the years to come. To validate their ultimate utility, it will be imperative to embed such biomarkers as a fundamental part of large, multicenter, prospective, therapeutic trials, before and after treatment with immune checkpoint inhibitors. Furthermore, from a scientific perspective, it will be of great value to understand the molecular determinants that mediate potent immune responses in a subset of HCCs, and whether therapeutic manipulation of these factors can switch an immunologically-inert tumor to an immunologically-active phenotype with ensuing clinical benefit.

\section{Acknowledgements}

None.

\section{Footnote}

Conflicts of Interest: JJ Harding has received consulting fees from Bristol-Myers Squib, Eisai, CytomX, and Eli Lilly and research funds from Bristol-Myers Squib. JC Osorio has no conflicts of interest to declare.

\section{References}

1. Kim HD, Song GW, Park S, et al. Association Between Expression Level of PD1 by Tumor-Infiltrating CD8+ T Cells and Features of Hepatocellular Carcinoma. Gastroenterology 2018;155:1936-50.e17.

2. Zhu AX, Finn RS, Edeline J, et al. Pembrolizumab in patients with advanced hepatocellular carcinoma previously treated with sorafenib (KEYNOTE-224): a non-randomised, open-label phase 2 trial. Lancet Oncol 2018;19:940-52.

3. El-Khoueiry AB, Sangro B, Yau T, et al. Nivolumab in patients with advanced hepatocellular carcinoma (CheckMate 040): an open-label, non-comparative, phase $1 / 2$ dose escalation and expansion trial. Lancet 2017;389:2492-502.

4. Harding JJ, El Dika I, Abou-Alfa GK. Immunotherapy in hepatocellular carcinoma: Primed to make a difference? Cancer 2016;122:367-77.

5. Cancer Genome Atlas Research Network, Cancer Genome Atlas Research Network. Comprehensive and Integrative Genomic Characterization of Hepatocellular Carcinoma. Cell 2017;169:1327-41.e23.

6. Zheng C, Zheng L, Yoo JK, et al. Landscape of Infiltrating $\mathrm{T}$ Cells in Liver Cancer Revealed by Single-Cell Sequencing. Cell 2017;169:1342-56.e16.

7. Sia D, Jiao Y, Martinez-Quetglas I, et al. Identification of an Immune-specific Class of Hepatocellular Carcinoma, Based on Molecular Features. Gastroenterology 2017;153:812-26.

8. Wang X, Teng F, Kong L, et al. PD-L1 expression in human cancers and its association with clinical outcomes. Onco Targets Ther 2016;9:5023-39.

9. Topalian SL, Hodi FS, Brahmer JR, et al. Safety, activity, and immune correlates of anti-PD-1 antibody in cancer. N Engl J Med 2012;366:2443-54.

10. Topalian SL, Taube JM, Anders RA, et al. Mechanismdriven biomarkers to guide immune checkpoint blockade in cancer therapy. Nat Rev Cancer 2016;16:275-87.

11. Cristescu R, Mogg R, Ayers M, et al. Pan-tumor genomic biomarkers for PD-1 checkpoint blockade-based immunotherapy. Science 2018;362(6411).

12. Snyder A, Makarov V, Merghoub T, et al. Genetic basis for clinical response to CTLA-4 blockade in melanoma. N Engl J Med 2014;371:2189-99.

13. Hellmann MD, Nathanson T, Rizvi H, et al. Genomic Features of Response to Combination Immunotherapy in Patients with Advanced Non-Small-Cell Lung Cancer. Cancer Cell 2018;33:843-52.e4.

14. Rizvi NA, Hellmann MD, Snyder A, et al. Cancer immunology. Mutational landscape determines sensitivity to PD-1 blockade in non-small cell lung cancer. Science 2015;348:124-8.

15. Harding JJ, Nandakumar S, Armenia J, et al. Prospective Genotyping of Hepatocellular Carcinoma: Clinical Implications of Next Generation Sequencing for Matching Patients to Targeted and Immune Therapies. Clin Cancer Res 2018. [Epub ahead of print].

16. Chowell D, Morris LGT, Grigg CM, et al. Patient HLA class I genotype influences cancer response to checkpoint blockade immunotherapy. Science 2018;359:582-7.

17. Zhou G, Sprengers D, Boor PPC, et al. Antibodies Against 
Immune Checkpoint Molecules Restore Functions of Tumor-Infiltrating T Cells in Hepatocellular Carcinomas. Gastroenterology 2017;153:1107-19.e10.

18. Li H, Wu K, Tao K, et al. Tim-3/galectin-9 signaling pathway mediates $\mathrm{T}$-cell dysfunction and predicts poor prognosis in patients with hepatitis B virus-associated hepatocellular carcinoma. Hepatology 2012;56:1342-51.

19. Inada Y, Mizukoshi E, Seike T, et al. Characteristics of immune response to tumor-associated antigens and immune cell profile in hepatocellular carcinoma patients. Hepatology 2018. [Epub ahead of print].

20. Kelley RK, Abou-Alfa GK, Bendell JC, et al. Phase I/II study of durvalumab and tremelimumab in patients with

doi: $10.21037 / \operatorname{tgh} .2018 .12 .04$

Cite this article as: Osorio JC, Harding JJ. Understanding and quantifying the immune microenvironment in hepatocellular carcinoma. Transl Gastroenterol Hepatol 2018;3:107. unresectable hepatocellular carcinoma (HCC): Phase I safety and efficacy analyses. J Clin Oncol 2017;35:4073.

21. Stein S, Pishvaian MJ, Lee MS, et al. Safety and clinical activity of $1 \mathrm{~L}$ atezolizumab + bevacizumab in a phase $\mathrm{Ib}$ study in hepatocellular carcinoma (HCC). J Clin Oncol 2018;36:4074.

22. Harding JJ, Erinjeri JP, Tan BR, et al. A multicenter pilot study of nivolumab (NIVO) with drug eluting bead transarterial chemoembolization (deb-TACE) in patients (pts) with liver limited hepatocellular carcinoma (HCC). J Clin Oncol 2018;36. doi: 10.1200/JCO.2018.36.15_suppl. TPS4146 